\title{
The clinical and genetic features of patients with hyper-immunoglobulin D syndrome (HIDS)
}

\author{
Betul Sozeri ${ }^{1 *}$, Ozlem Cam ${ }^{2}$, Metin Delebe ${ }^{2}$, Sevgi Mir ${ }^{1}$, Afig Berdeli ${ }^{3}$ \\ From 21st European Pediatric Rheumatology (PReS) Congress \\ Belgrade, Serbia. 17-21 September 2014
}

\begin{abstract}
Introduction
Mevalonate kinase deficiency (MKD) is a rare autosomal recessive disorder causing 1 of 2 phenotypes, hyperimmunoglobulin D syndrome and mevalonic aciduria, presenting with recurrent fever episodes, often starting in infancy, and sometimes evoked by stress or vaccinations. This autoinflammatory disease is caused by mutations encoding the mevalonate kinase (MVK) gene and is classified in the group of periodic fever syndromes. HIDS is characterized by recurrent fever attacks of 3-7 days that begin in infancy and recur every 4-6 weeks. The febrile period is accompanied by lymphadenopathy, arthralgia, abdominal pain, diarrhea, aphthous ulcers, and varying degree of skin involvement. The course and severity of the disease may be quite different. There is no effective or proven therapy for HIDS.
\end{abstract}

\section{Objectives}

We aim to determine the clinical and genetic characteristics together with the underlying MVK genotypes in single center during a period of 2 years.

\section{Methods}

A retrospective review of medical records for patients referred for HIDS over 2 years. We obtain 40 patients (22 female, 18 male) and 25 healthy controls.

\section{Results}

The median age of first attack was 33 months (range 2-62 months). The median age of diagnosis was 60 months. The most common symptoms of high fever accompanied respectively: lymphadenopathy $(\mathrm{n}=27)$, abdominal pain $(\mathrm{n}=26)$, arthralgia $(\mathrm{n}=20)$, diarrhea $(\mathrm{n}=15)$, aphthous stomatitis $(n=13)$, vomiting $(n=12)$ and maculopapuler rash $(n=6)$ were determined. Amyloidosis was found in a patient $(0.3 \%)$. The mean serum IgD level was $129 \pm 92$ $\mathrm{mg} / \mathrm{dl}$, it was be normal in $43 \%$ of patients.

We found mutations in $75 \%$ of patients $(\mathrm{n}=30)$ in exon 3 and 11 (c155G>A; c1129G>A) in MVK gene. We applied colchicine therapy in $40 \%$ of patients while intermittent steroid therapy $20 \%$ of patients. Empiric colchicine (40\%) and glucocorticosteroids (20\%) controlled flares in majority of patients with HIDS. Three patients had also tonsillectomy. We used biologic therapy in 3 patients (canacinumab $(\mathrm{n}=2)$, anakinra $(\mathrm{n}=1)$ ).

\section{Conclusion}

In conclusion, HIDS is characterized with early onset an autoinflammatory disease and may to result frequent and uncontrolled attacks.

\section{Disclosure of interest}

None declared.

\section{Authors' details}

${ }^{1}$ Pediatric Rheumatology, Ege University Faculty of Medicine, Izmir, Turkey. ${ }^{2}$ Pediatrics, Ege University Faculty of Medicine, Izmir, Turkey. ${ }^{3}$ Moleculer Genetic, Ege University Faculty of Medicine, Izmir, Turkey.

Published: 17 September 2014

doi:10.1186/1546-0096-12-S1-P257

Cite this article as: Sozeri et al:: The clinical and genetic features of patients with hyper-immunoglobulin D syndrome (HIDS). Pediatric

Rheumatology 2014 12(Suppl 1):P257. 